\title{
Trends in mortality from respiratory system diseases in Greece during the financial crisis
}

To the Editor:

As a result of the ongoing economic recession in Greece, gross domestic product and health expenditure declined by $25 \%$, unemployment increased from 9.6 to $26.5 \%$ [1], while unmet needs for medical care increased between 2009 and 2014 [2, 3]. Among others, the decline in disposable income has transformed cigarettes into luxury goods [4], tobacco consumption has declined sharply [5-7], sales and use of private vehicles have declined $[1,5]$ and low economic and industrial activity has contributed to the decline in concentrations of major air pollutants in urban areas, such as Athens [8].

Greece provides a unique opportunity to study the population impact of the opposing influence of deteriorating health services and socioeconomic conditions on the one hand, and improving behavioural and environmental factors on the other. No study has explored the trends in respiratory diseases that constitute the third most frequent cause of death in Greece, following cardiovascular disease and cancer [1], and are heavily influenced by smoking and air quality. The aim of this study was to assess the short-term effects of healthcare, behavioural and environmental changes in Greece during the financial crisis on mortality from respiratory diseases.

Data on the estimated population and number of deaths by cause were obtained from the Hellenic Statistical Authority (ELSTAT; Piraeus, Greece) [1] for 2001 to 2013. Deaths were reported by ELSTAT in groups of causes from the ninth version of the International Classification of Diseases (ICD-9). We used data on disease codes 460-519 (all diseases of the respiratory system) and codes 160-165 (malignant neoplasms of respiratory and intrathoracic organs). The number of deaths by individual ICD-9 code was not available.

Mortality was directly standardised by sex and five-year age group, with the population of Greece in 2009 used as the standard population. We defined 2010 as the year that the crisis "started", as it was in early 2010 that austerity measures were first introduced $[2,3]$. We calculated the average annual change (\%) in standardised mortality for each disease group before (2001-2009) and during the crisis (2010-2013). We also compared the expected, based on underlying trends, and actual mortality during the crisis to estimate the number of deaths from diseases of the respiratory system avoided during the crisis. Crude mortality rates by age group (0-14; $15-24 ; 25-34 ; 35-44 ; 45-54 ; 55-64 ; 65-74$; and $>74$ years) were also calculated. We conducted an interrupted time series (ITS) analysis, which compared the trend (slope) in mortality before and during the crisis; $\mathrm{p}<0.05$ indicated a statistically significant difference between the slopes before and during the crisis. All analyses were conducted using Stata 14 for Windows (StataCorp LP, College Station, TX, USA).

A total of 10974 deaths (9.8\% of all deaths) in 2013 were attributed to diseases of the respiratory system (figure 1a). Malignant neoplasms of respiratory and intrathoracic organs caused $6.6 \%$ of total deaths (figure $1 \mathrm{~b}$ ). Standardised mortality from all diseases of the respiratory system decreased at an average rate of 3.2\% per year during the crisis (97.1 per 100000 in 2009; 84.9 per 100000 in 2013), in contrast to an average annual increase of 3.3\% in the pre-crisis period (75.7 per 100000 in 2001; 97.1 per 100000 in 2009). The ITS analysis revealed a significant change in the trend during the crisis $(p=0.01)$. The trends for respiratory and intrathoracic cancers were quite similar before and during the crisis (average annual increase $0.2 \%$ versus $0.6 \%$ respectively; $\mathrm{p}=0.196$ ).

Crude mortality from diseases of the respiratory system increased between 2001 and 2009 and decreased after 2009 across all age groups, but the change in slope was significant only in the 45-54, 55-64 and 65-74 age groups. On the contrary, there was no clear trend in crude mortality from malignant neoplasms. Comparing the expected with actual mortality during the crisis, we estimated that, between 2010 and 2013, there were 8202 fewer deaths from diseases of the respiratory system.

Mortality from respiratory diseases and cancers of the respiratory system can be influenced by the prevalence and intensity of smoking [9] and levels of air pollution [10], but it can also be dependent on the availability of effective treatments, early diagnosis, adherence to appropriate medication and other healthcare-related factors. 


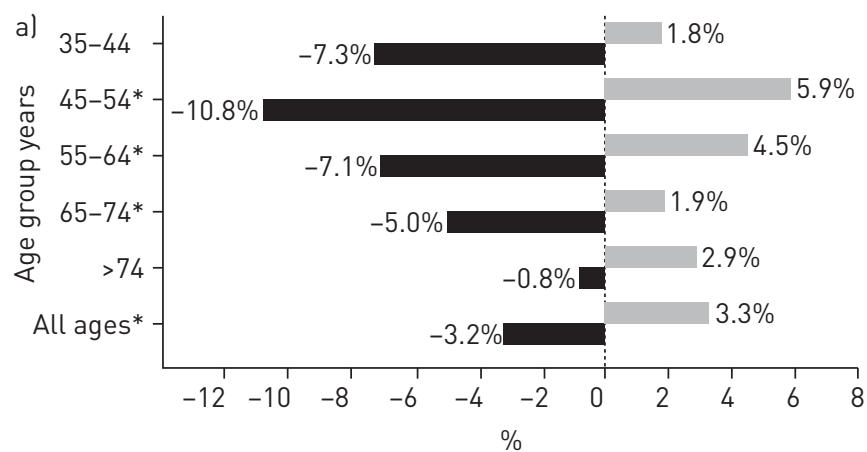

Average annual change in crude mortality 2001-2009

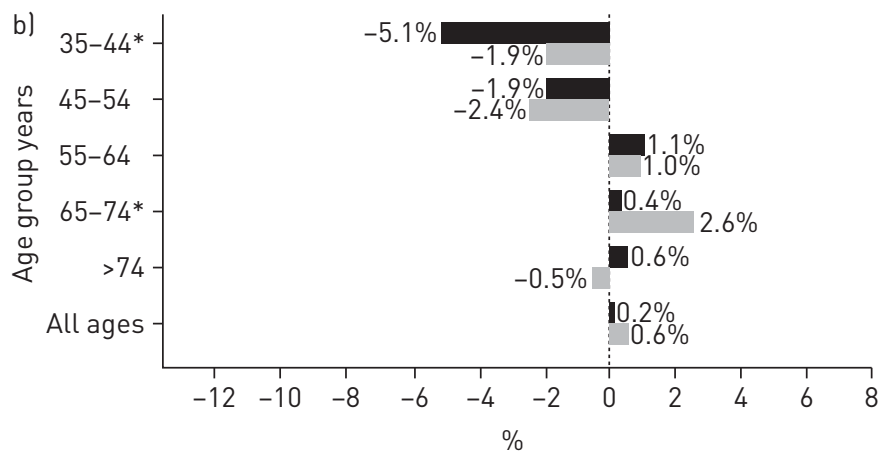

Average annual change in crude mortality 2010-2013

FIGURE 1 Average changes in crude mortality by age group (per 100000) in Greece, 2001-2009 and 2010-2013 for a) diseases of the respiratory system and b) malignant neoplasms of respiratory and intrathoracic organs. *: $p<0.05$ in the interrupted time series analysis; it denotes a statistically significant change in the slope before and during the crisis. Mortality rates for all ages are standardised by sex and five-year age groups. Crude mortality is not presented for age groups in which a very small number of deaths was reported (i.e. $<3$ per 100000 ).

The prevalence of smoking declined from 42 to $38 \%$ [7] and cigarette consumption by 37.5\% between 2009 and 2014 [6], a change mostly achieved within the first couple of years [5]. As a result, the overall population exposure to smoking during the crisis has been much lower than before. However, the implementation of existing smoke-free legislation has been inconsistent and the majority of the population is regularly exposed to second-hand smoke [11]. The concentrations of pollutants, such as particles with a $50 \%$ cut-off aerodynamic diameter of 2.5 and $10 \mu \mathrm{m}$, and sulfate, have declined rapidly in Athens [8], despite the fact that biomass consumption for heating purposes has had negative effects on air pollution during the cold winter months $[8,12]$. Data for other urban areas are probably similar to Athens, where $40 \%$ of the population resides. Thus, improvements in overall air quality are likely to have an important effect at the population level.

However, the provision of healthcare has deteriorated, as a result of budget cuts and the introduction of user fees and co-payments. A large number of people face difficulties accessing essential health services or paying for their medication [2,3], which could lead to delays in diagnosing or treating diseases. Problems with treating patients with chronic obstructive pulmonary disease (COPD) and lung cancer have been reported $[13,14]$, possibly reflecting the strains on the Greek healthcare system.

It seems that the balance of these opposing forces has been different between the two main groups of respiratory diseases, as mortality from diseases of the respiratory system declined while the trend in mortality from malignancies did not change during the crisis. Some of the nonmalignant respiratory diseases, such as COPD, are more sensitive to short-term changes in smoking and air pollution, compared to cancer which has a longer natural history. We have no data regarding the potentially differential effects of the crisis on the treatment of different diseases. Therefore, it seems that, at least for nonmalignant diseases of the respiratory system, environmental and behavioural factors have played a more important role compared to healthcare. The differences in mortality trends by age support this hypothesis, as younger groups have benefited more during the crisis. Older people may rely more on the healthcare system, thus experiencing the negative effects of deteriorating healthcare in the country. Moreover, the prevalence of smoking was already quite low among older age groups before the crisis and has not changed much in recent years [5].

It is important to note that none of the favourable changes in smoking and air quality has been a result of government-led policies [11]. Even though causal interpretations of our findings should be made with caution, the austerity measures may have had unintended positive consequences in some key exposures, but there is no evidence that these trends can be sustained in the future. In any case, smoke-free environment laws can have a significant impact on morbidity and mortality within a very short period of time, producing savings in healthcare costs [15]. Our findings show the huge potential of environmental and behavioural factors in reducing mortality from respiratory diseases, even when health services are deteriorating. This is particularly important, not only for Greece, but for any country in which healthcare is under pressure. Focusing on prevention and environmental exposure can have a great impact, not only by preventing disease, but also by alleviating the pressure on healthcare services, thus improving care for existing patients. 
Filippos T. Filippidis $\odot^{1}$, Vasiliki Gerovasili ${ }^{2}$, William D-C. Man $^{3}$ and Jennifer K. Quint ${ }^{4}$

${ }^{1}$ Dept of Primary Care and Public Health, School of Public Health, Imperial College London, London, UK. ${ }^{2}$ Dept of Respiratory Medicine, Harefield Hospital, Royal Brompton \& Harefield NHS Foundation Trust, London, UK. ${ }^{3}$ NIHR Respiratory Biomedical Research Unit, Royal Brompton \& Harefield NHS Foundation Trust and Imperial College London, London, UK. ${ }^{4}$ Dept of Respiratory Epidemiology, Occupational Medicine and Public Health, National Heart and Lung Institute, Imperial College London, London, UK.

Correspondence: Filippos T. Filippidis, Dept of Primary Care and Public Health, Imperial College London, The Reynolds Building, St Dunstan’s Road, London W6 8RP, UK. E-mail: f.filippidis@imperial.ac.uk

Received: April 182016 | Accepted after revision: Aug 02 2016 | First published online: Oct 062016

Conflict of interest: None declared.

\section{References}

$1 \quad$ Hellenic Statistical Authority. Statistics 2016. www.statistics.gr Date last accessed: January 28, 2016.

2 Simou E, Koutsogeorgou E. Effects of the economic crisis on health and healthcare in Greece in the literature from 2009 to 2013: a systematic review. Health Policy 2014; 115: 111-119.

3 Kentikelenis A, Karanikolos M, Reeves A, et al. Greece's health crisis: from austerity to denialism. Lancet 2014 383: 748-753.

4 Tarantilis F, Athanasakis K, Zavras D, et al. Estimates of price and income elasticity in Greece. Greek debt crisis transforming cigarettes into a luxury good: an econometric approach. BMJ Open 2015; 5: e004748.

5 Filippidis FT, Schoretsaniti S, Dimitrakaki C, et al. Trends in cardiovascular risk factors in Greece before and during the financial crisis: the impact of social disparities. Eur J Public Health 2014; 24: 974-979.

6 Euromonitor International. Passport Database 2016. http://go.euromonitor.com/passport.html.

7 European Commission. Special Eurobarometer 429. Attitudes of Europeans towards tobacco and electronic cigarettes. http://ec.europa.eu/public_opinion/archives/ebs/ebs_429_en.pdf.

8 Paraskevopoulou D, Liakakou E, Gerasopoulos E, et al. Sources of atmospheric aerosol from long-term measurements (5 years) of chemical composition in Athens, Greece. Sci Total Environ 2015; 527-528: 165-178

9 U.S. Department of Health and Human Services. The Health Consequences of Smoking-50 Years of Progress. A Report of the Surgeon General, 2014. http://www.surgeongeneral.gov/library/reports/50-years-of-progress/.

10 Hoek G, Krishnan RM, Beelen R, et al. Long-term air pollution exposure and cardio-respiratory mortality: a review. Environ Health 2013; 12: 43

11 Filippidis FT, Tzoulaki I. Greece giving up on tobacco control. Addiction 2016; 111: 1306-1307.

12 Manoli E, Kouras A, Karagkiozidou O, et al. Polycyclic aromatic hydrocarbons (PAHs) at traffic and urban background sites of northern Greece: source apportionment of ambient PAH levels and PAH-induced lung cancer risk. Environ Sci Pollut Res Int 2016; 23: 3556-3568.

13 Tsiligianni IG. COPD patients trapped in the financial crisis in rural Crete. Lancet Respir Med 2013; 1: e31-e32.

14 Bakogeorgos M, Mountzios G, Bournakis E, et al. Do elderly patients with non-small cell lung cancer get the best out of recent advances in first-line treatment? A comparative study in two tertiary cancer centers in Greece. J Geriatr Oncol 2015; 6: 111-118.

15 Kalkhoran S, Glantz SA. Smoke-free policies: cleaning the air with money to spare. Lancet 2014; 383: 1526-1528.

\section{Acromegaly in sleep apnoea patients: a large observational study of 755 patients}

\section{To the Editor:}

Acromegaly is a rare, chronic and progressive disease characterised by excess secretion of growth hormone with raised insulin-like growth factor I (IGF-I) levels and usually caused by a pituitary adenoma $[1,2]$. Its prevalence is estimated at $40-480$ cases per million, depending on the study $[1,3]$. The diagnosis of acromegaly, frequently made late because of the insidious nature of the disease, is generally based on symptoms of excess growth hormone, such as acral enlargement, soft-tissue swelling, arthralgia, jaw prognathism, hyperhidrosis, osteoarthritis and frontal bossing, or symptoms of a pituitary adenoma, such as headaches, visual defects or pituitary insufficiency. Owing to prolonged untreated progression of the condition, patients often exhibit established systemic complications at diagnosis, such as diabetes mellitus (19$56 \%$ ) [2], hypertension (30-40\%) and respiratory/cardiac failure $(60 \%)$ [4, 5], which are the main determinants of prognosis and premature mortality [1]. The majority of new diagnoses of acromegaly are detected by primary care physicians, or specialists other than endocrinologists. The therapeutic options for acromegaly include endoscopic surgery, medical therapies (long-acting somatostatin analogues; dopamine agonists; growth hormone receptor antagonists) and pituitary radiotherapy [6, 7]. 45-80\% of acromegaly 